\title{
Towards an improved early diagnosis of neurodegenerative diseases: the emerging role of in vitro conversion assays for protein amyloids
}

\author{
Niccolò Candelise ${ }^{1}$, Simone Baiardi ${ }^{2,3}$, Alessia Franceschini ${ }^{2}$, Marcello Rossi ${ }^{2}$ and Piero Parchi ${ }^{1,2^{*}}$ (D)
}

\begin{abstract}
Tissue accumulation of abnormal aggregates of amyloidogenic proteins such as prion protein, a-synuclein, and tau represents the hallmark of most common neurodegenerative disorders and precedes the onset of symptoms by years. As a consequence, the sensitive and specific detection of abnormal forms of these proteins in patients' accessible tissues or fluids as biomarkers may have a significant impact on the clinical diagnosis of these disorders. By exploiting seeded polymerization propagation mechanisms to obtain cell-free reactions that allow highly amplified detection of these amyloid proteins, novel emerging in vitro techniques, such as the real-time quakinginduced conversion assay (RT-QulC) have paved the way towards this important goal. Given its high accuracy in identifying misfolded forms of prion protein from Creutzfeldt-Jakob disease (CJD) CSF, RT-QulC has already been included in the diagnostic criteria for the clinical diagnosis of sporadic CJD, the most common human prion disease. By showing that this assay may also accurately discriminate between Lewy body disorders and other forms of parkinsonisms or dementias, more recent studies strongly suggested that CSF RT-QuIC can also be successfully applied to synucleinopathies. Finally, preliminary encouraging data also suggested that CSF RT-QulC might also work for tau protein, and accurately distinguish between 3R- and 4R tauopathies, including Pick's disease, progressive supranuclear palsy, and corticobasal degeneration. Here we will review the state of the art of cell-free aggregation assays, their current diagnostic value and putative limitations, and the future perspectives for their expanded use in clinical practice.
\end{abstract}

Keywords: RT-QulC, Biomarker, Diagnosis, Prion disease, Parkinson's disease, Alzheimer's disease, Lewy bodies, Prion protein, a-Synuclein, Tau

\footnotetext{
* Correspondence: piero.parchi@unibo.it

'Department of Experimental, Diagnostic and Specialty Medicine (DIMES), University of Bologna, Bologna, Italy

${ }^{2}$ RCCS, Istituto delle Scienze Neurologiche di Bologna, Ospedale Bellaria, Via Altura 1/8, 40139 Bologna, Italy

Full list of author information is available at the end of the article
}

(c) The Author(s). 2020 Open Access This article is licensed under a Creative Commons Attribution 4.0 International License, which permits use, sharing, adaptation, distribution and reproduction in any medium or format, as long as you give appropriate credit to the original author(s) and the source, provide a link to the Creative Commons licence, and indicate if changes were made. The images or other third party material in this article are included in the article's Creative Commons licence, unless indicated otherwise in a credit line to the material. If material is not included in the article's Creative Commons licence and your intended use is not permitted by statutory regulation or exceeds the permitted use, you will need to obtain permission directly from the copyright holder. To view a copy of this licence, visit http://creativecommons.org/licenses/by/4.0/ The Creative Commons Public Domain Dedication waiver (http://creativecommons.org/publicdomain/zero/1.0/) applies to the data made available in this article, unless otherwise stated in a credit line to the data. 


\section{Introduction}

The most common neurodegenerative diseases feature protein misfolding, aggregation, and accumulation of protein amyloids inside or outside cells [17]. Consequently, the detection of these abnormal proteins in biofluids and accessible tissues represents the ideal candidate for the early antemortem identification of these disorders. However, owing to considerable differences in their concentration among various tissues and fluids, these protein aggregates are, in most cases, readily detectable by standard assays only in the affected brain. Thus, apart from Alzheimer's disease (AD), in which changes in the CSF levels of certain tau and amyloidbeta isoforms reflect the ongoing deposition of these proteins in the brain, surrogate markers of neuronal damage such as 14-3-3, total tau and neurofilament proteins represented the only available biofluid markers to support the clinical diagnosis of neurodegenerative diseases until recently [85]. Although advancing ultrasensitive immunoassays, enabling the detection of single molecules of the target of interest, allow the measurement of some of these markers also in the blood, making them a reliable tool for population screening, prognostic assessment, and monitoring of drug effect in clinical trials [3], the search for disease-specific markers remains the primary goal in the field. In this context, the recent development of ultrasensitive assays that indirectly reveal minute amounts of misfolded prion protein and other prion-like proteins, based on an amplification strategy, represented a major breakthrough in the field with enormous diagnostics potential for neurodegenerative diseases. In this work, we will review the state of the art of cell-free conversion assays and their current and foreseeable diagnostic applications in clinical practice. In particular, we will focus on the most promising of these assays, the real-time quaking-induced conversion (RT$\mathrm{QuIC}$ ), which uses a recombinant protein as substrate, intermittent shaking to foster conversion, and Thioflavin- $\mathrm{T}$ (ThT) to monitor the aggregation phase in real-time (Fig. 1) [91].

\section{Main text}

\section{Search strategy and selection criteria}

We searched Google Scholar and PubMed using the terms "prion", "Creutzfeldt-Jakob disease", "alfa-synuclein”, "tau protein", "parkinsonism”, "Lewy body”, "tauopathy", "Parkinson's disease", "MSA", "PSP”, "CBD”, "AD" and "Pick's disease", each in combination with "real time-quaking-induced conversion" or "RT-QuIC" or "PMCA". Studies and articles written in English were selected based on our rating of the methodological aspects and of their relevance to the diagnosis of human neurodegenerative diseases. A few articles providing reference to basic concepts or general information were added based on personal knowledge of the scientific literature.

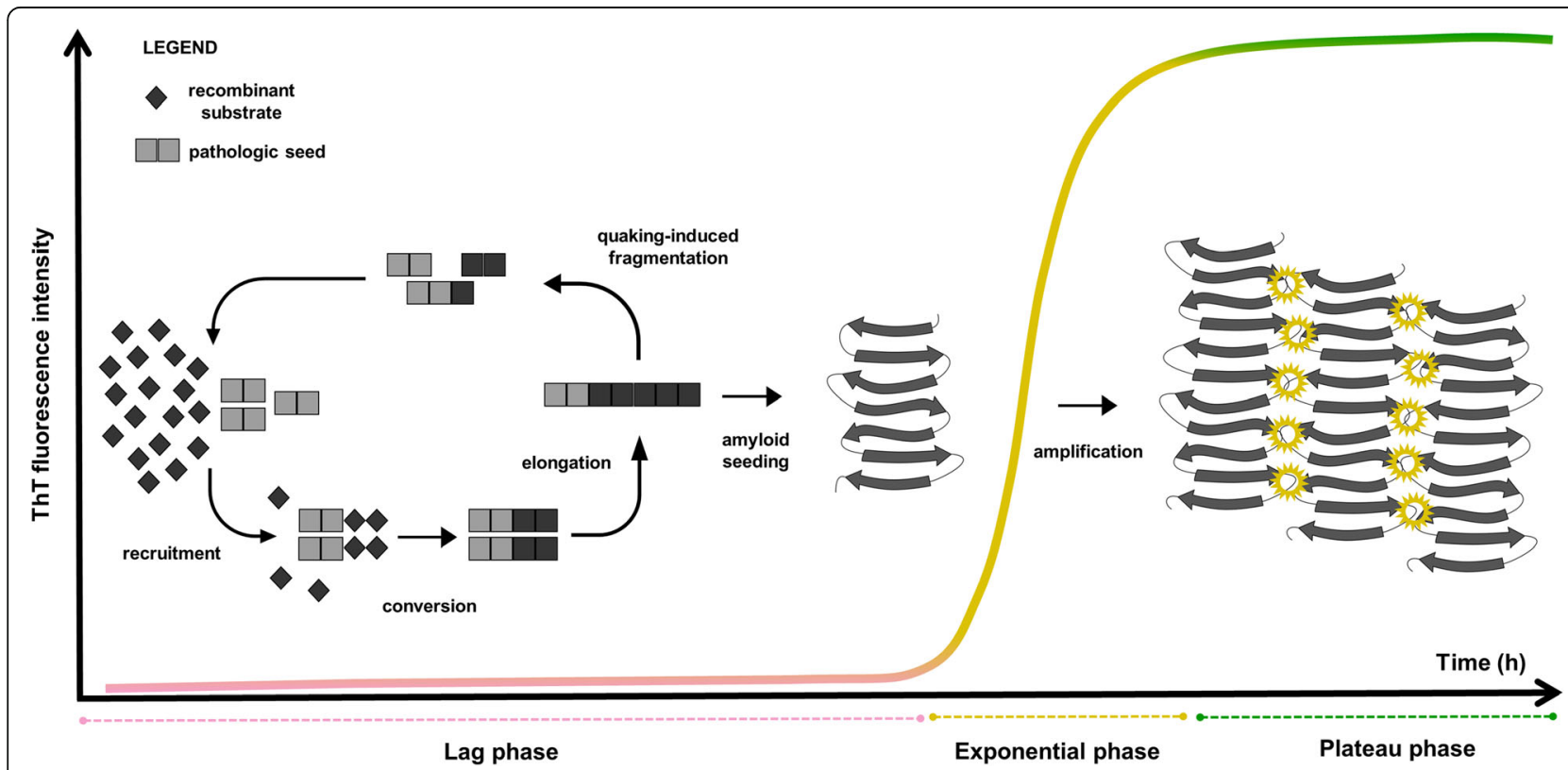

Fig. 1 Schematic representation of the RT-QulC reaction. The RT-QulC reaction may be divided into temporal stages: the lag phase, the exponential phase and the plateau phase. The lag phase represents the time required for the reaction to take place. During this step, the seed is allowed to contact the substrate and to trigger the conformational change. Once enough energy is received by the system, ThT sensitive oligomers emerge, which incorporate the monomers into small aggregates (exponential phase). Eventually, when all the substrate is incorporated into fibrils, a plateau phase is observed 


\section{A brief history of in vitro conversion assays}

The concept of cell-free aggregation assays stems from the discovery of the protein-only mechanism for the propagation of the prion protein $(\operatorname{PrP})$ as the sole pathogenic agent of transmissible spongiform encephalopathies including CJD and other human and animal prion diseases, such as scrapie in sheep, and chronic wasting disease in cervids [68, 69]. Aggregation of $\operatorname{PrP}$ was found to be dependent on the secondary structure's conformational changes, switching from $\alpha$-helix enriched, as in the cellular prion protein, $\operatorname{PrP}^{\mathrm{C}}$, into a $\beta$-sheet enriched conformation as in the abnormal scrapie prion protein, $\operatorname{PrP}^{\mathrm{Sc}}[8,15]$. The modification of the secondary structure allows the misfolded protein to contact and convert its native counterpart, causing a positive feedback that eventually leads to aggregates and amyloid fibrils [70]. Moreover, biochemical features, such as protease resistance, detergent solubility, and thermal stability, are dramatically increased in the misfolded forms compared to the native protein $[56,77]$.

In the attempt to better understand $\operatorname{PrP}$ conversion, in the mid '90s, Kocisko and co-workers successfully generated aggregated species of $\operatorname{PrP}$ in a cell-free assay [47]. In this work, an excess of $\mathrm{PrP}^{\mathrm{Sc}}$ isolated from prion-infected hamsters was partially denatured in guanidine- $\mathrm{HCl}$ and incubated with 35S-labelled recombinant $\operatorname{PrP}^{C}$ derived from uninfected hamster's brain. The demonstrations of proteinase $\mathrm{K}(\mathrm{PK})$ resistance labeled $\operatorname{PrP}$ confirmed the de novo formation of misfolded species.

As a significant technical implementation to this assay, Saborio and co-workers [76] introduced cyclical sonication and incubation steps, which allowed to speed up the in vitro conversion and amplify as little as attograms of $\mathrm{PrP}^{\mathrm{Sc}}$ in the presence of an excess of healthy brain homogenate as a source of $\operatorname{PrP}^{\mathrm{C}}$. In this assay, termed protein misfolding cyclic amplification (PMCA), sonication provided the energy required to break aggregated species of PrP into misfolded, aggregation-competent species, while incubation allowed the conversion of the monomers into their pathogenic form. Through various sonication and incubation passages, the researchers were able to amplify the original $\operatorname{PrP}^{\mathrm{Sc}}$ seeds greatly. As in the original assay, the detection relied on partial digestion with PK, followed by western blotting using specific $\operatorname{PrP}$ antibodies. Strikingly, PMCA was able to detect the presence of $\mathrm{PrP}^{\mathrm{Sc}}$ in the brain of pre-symptomatic hamsters [86], rendering this method fundamental for both research and diagnostic applications. However, a significant limitation of the PMCA was that the end-products would still retain their infectiousness, posing a problem for the application in routine clinical practice. Moreover, PMCA appeared to be sensitive to contaminants, resulting in a suboptimal reproducibility $[9,20]$.
The use of bacterially expressed recombinant $\operatorname{PrP}$ as reaction substrate overcame the need for brain homogenates as the source of $\operatorname{PrP}^{\mathrm{C}}$ [4], allowing the usage of a vast excess of the substrate facilitating the conversion into its pathogenic counterpart.

Methodological improvement of the aggregation assay included the swap of sonication with automated shaking, defined as quaking-induced conversion (QuIC). In addition to the clear advantages of an automated system, with this novel methodology, Atarashi and co-workers [4] were able to improve reproducibility compared to PMCA and shorten the time required to form further aggregates.

In parallel, Colby and co-workers [18] developed an assay for the detection of protein aggregates termed amyloid seeding assay, which relied on ThT for the detection of aggregate formation, thus bypassing the laborious and time-consuming detection by immunoblot. This fluorescent dye shows enhanced emission and a shift toward the red spectrum when bound to amyloid structures [10]. The scientists used purified mouse recombinant prion protein as the substrate for the seeded aggregation of either preformed fibrils derived from the same mouse PrP, brain homogenates from PrP infected mice, or CJD brain material, yielding a real-time rapid and ultra-sensitive (within attograms) detection of prions.

By combining the PMCA and QuIC cyclic amplification with the real-time detection of the products by ThT, Wilham and co-workers [91] developed the first version of the RT-QuIC, in which minute amounts of seed derived from brain homogenates were sufficient to trigger the aggregation of an excess of the recombinant prion protein, monitored in real-time by ThT (Fig. 1). The methodology was further improved to detect PrP seeds in the CSF [5], proving that the RTQuIC could have a significant impact in the clinical practice.

From here, several studies tested the RT-QuIC for its diagnostic accuracy in prion disease and other neurodegenerative conditions. Nowadays, the diagnostic criteria for sporadic CJD (sCJD) already include RT-QuIC [94], and efforts are ongoing to expand the RT-QuIC application to the detection of other aggregation-prone proteins involved in the pathogenesis of neurodegenerative diseases.

In the next sections, we will review the results with clinical diagnostic implications obtained to date with the three main RT-QuIC assays thus far developed, namely PrP RT-QuIC, alfa-synuclein ( $\alpha$-syn) RTQuIC, and tau RT-QuIC (Fig. 2). A few studies on the so-called $\alpha$-syn PMCA, performed with a protocol adapted to the same basic principles of the RT-QuIC, will also be reviewed. 


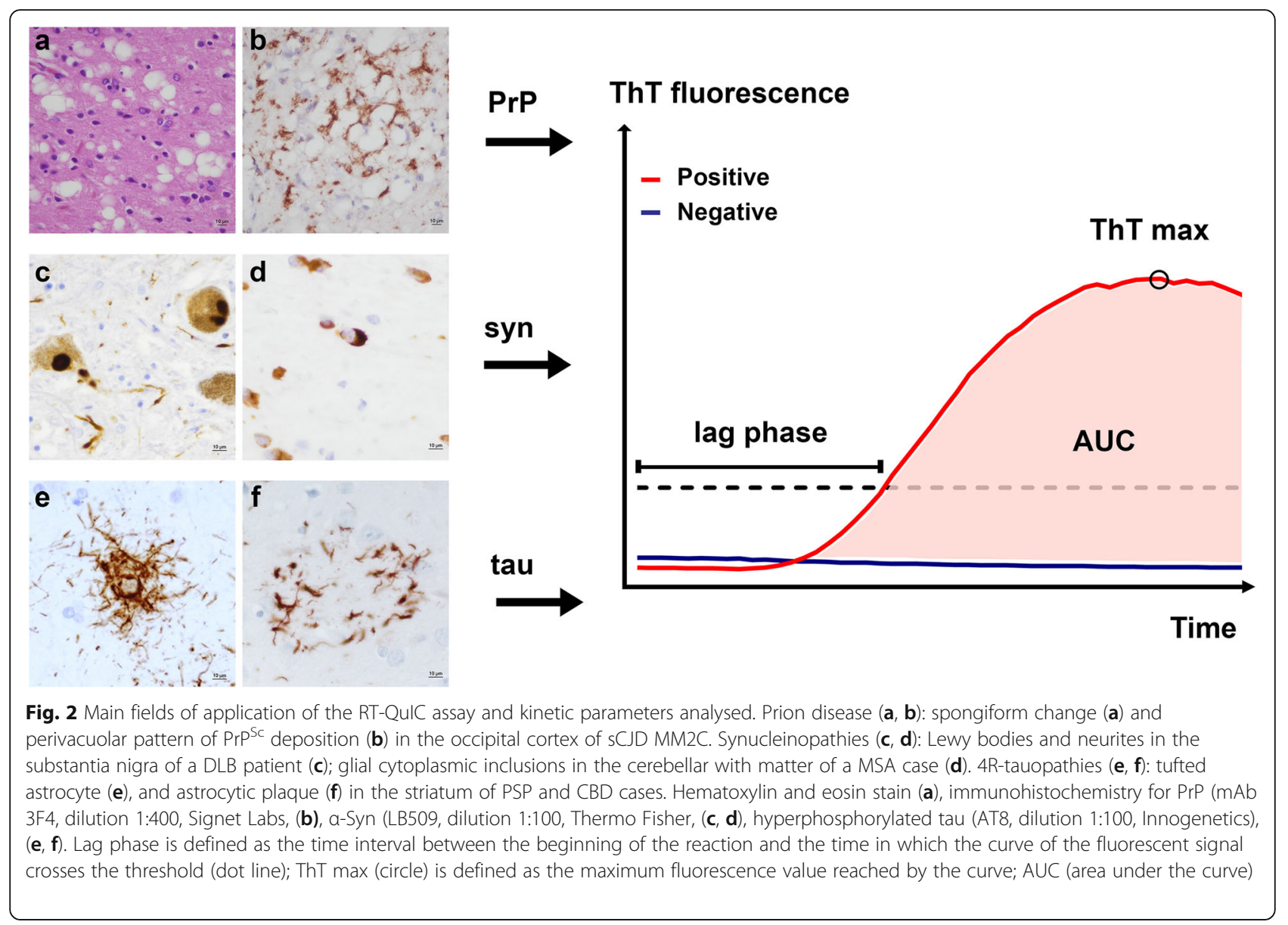

\section{Diagnostic value of PrP RT-QulC in rapidly progressive neurological syndromes}

Despite their low prevalence, prion diseases encompass a broad spectrum of clinicopathological variants with significant heterogeneity in disease duration, symptomatology, and type or regional distribution of brain lesions. Current classification of prion diseases includes four major phenotypic entities, namely Creutzfeldt-Jakob disease (CJD), Gerstmann-Sträussler-Scheinker disease (GSS), fatal insomnia (FI), and variably proteasesensitive prionopathy (VPSPr) [7] (Table 1).

Moreover, some of these groups include disease subtypes with distinguishing molecular and clinicopathological features. Most significantly, CJD, by far the most prevalent prion disease in humans, comprises six subtypes, which are primarily determined by the genotype at the polymorphic codon 129 (encoding methionine, M or valine, $\mathrm{V}$ ) in PRNP, and by the type (1 or 2 ) of abnormal prion protein $\left(\mathrm{PrP}^{\mathrm{Sc}}\right)$ accumulating in the brain (i.e., MM1, VV1, MM2, VV2, MV2) [63-65] (Table 1). The broad clinical heterogeneity at disease onset, which largely overlaps with that of other rapidly progressive dementias (RPDs), and the variable disease progression affecting survival times, significantly challenge the early diagnosis and clinical management of patients with prion disease [6]. Brain-derived CSF protein assays serving as surrogate markers for neuronal damage and diffusion-weighted magnetic resonance imaging (DWMRI) have provided the primary support for the clinical diagnosis of sporadic prion disease in the past 15-20 years. However, both biomarkers demonstrated an incomplete diagnostic accuracy with significant variability across disease subtypes. For example, in sCJD, DW-MRI may disclose a typical, almost subtype-specific, lesion pattern in a subset of cases, including a high signal intensity in the basal ganglia and thalamus in the VV2 subtype, a prominent, often isolated, cortical signal hyperintensity in subjects MM2C or VV1 or marked asymmetry of the signal hyperintensity in the MM1 variant [94]. On the other hand, the same investigation may be unrevealing in $5-10 \%$ of cases, depending on the disease subtype and the time from disease onset, and yields a variable percentage of false-positive scans in RPDs with non-prion etiologies [90]. According to most studies, abnormal signal hyperintensities on fluid-attenuated inversion recovery (FLAIR) or DW-MRI involve 91-96\% of SCJD cases and have an overall specificity of $87-98 \%$ $[14,75,84,90,92,93]$. However, diagnostic accuracy 
Table 1 Current classification of human prion diseases according to etiology and phenotypic features

\begin{tabular}{|c|c|c|}
\hline Diseases & Etiology & Phenotype $(s)^{a}$ \\
\hline \multirow[t]{3}{*}{ Creutzfeldt-Jakob disease } & Sporadic & $\begin{array}{l}\text { MM(V)1 (typical/myoclonic variant) } \\
\text { W2 (ataxic/cerebellar or Brownell-Oppenheimer variant) } \\
\text { MV2K (kuru-plaque variant) } \\
\text { MM(V)2C (cortical variant) } \\
\text { W1 (cortico-striatal variant) } \\
\text { Atypical MM1 with PrP-amyloid plaques in white matter }\end{array}$ \\
\hline & Genetic & According to PRNP haplotype (mutation + codon 129) and PrPSC type \\
\hline & Acquired & $\begin{array}{l}\text { I. latrogenic (MM1, W2, MV2K and MMiK }) \\
\text { II. Variant }\end{array}$ \\
\hline \multirow[t]{2}{*}{ Fatal Insomnia } & Sporadic & MM2T (thalamic variant) \\
\hline & Genetic & Fatal familial insomnia or MM(V)2T (thalamic variant) \\
\hline Gerstmann-Sträussler-Scheinker disease & Genetic & According to PRNP haplotype (mutation + codon 129) and size/s of PrPSC fragment/s \\
\hline Variably protease-sensitive prionopathy & Sporadic & According to PRNP codon 129 genotype and PK-resistance of PrPSc fragments \\
\hline
\end{tabular}

${ }^{a}$ The nomenclature and classification of sporadic Creutzfeldt-Jakob disease in distinct phenotypes is largely based on the combination of the genotype at codon 129 of PRNP (methionine, M, or valine, V) and the PrP ${ }^{\mathrm{SC}}$ type 1 or 2, as defined by the distinctive size ( 21 and $\left.19 \mathrm{kDa}\right)$ of their PK-resistant core

$\mathrm{b}_{\mathrm{i}}$ (=intermediate) refers to an electrophoretic mobility halfway between types 1 and $2 ; \mathrm{k}=$ with PrP-amyloid kuru plaques

was significantly higher in studies involving CJD surveillance centers than community hospitals [14]. In this regard, it is worth noting that CJD surveillance centers, likely as a consequence of improved technology and increased awareness of DW hyperintensities, have been witnessing an increase of referrals due to MRI findings of isolated cortical ribboning in patients with seizures, hyponatremia, Wernicke's encephalopathy or even ischemia, often in the absence of typical clinical features of CJD. To limit the occurrence of such false-positive results and favor specificity, only hyperintensities involving the striatum (caudate and putamen nuclei) or at least two neocortical regions in different lobes except for the frontal (or both), are considered "typical" for CJD by current diagnostic criteria [93]. By applying these restrictive criteria to a large patient cohort, a recent study showed a $96.5 \%$ specificity but only a $73.2 \%$ sensitivity for CJD diagnosis [11]. Of notice, however, when more permissive criteria were applied (i.e., an abnormal signal in at least one region among the cerebral cortex of all lobes, caudate, putamen, and thalamus), the authors did not report any loss in specificity (95.5\%) besides the expected increase in sensitiviy (92.7\%) [11].

Similarly, CSF assays based on surrogate markers such as protein $14-3-3$ and $t$-tau yield false-positive results in at least $10 \%$ of clinically selected cases (as for MRI, the erroneous use of these markers as a screening test in all RPDs increases this percentage significantly), with a wide diagnostic specificity range among studies (between 57 and 97\%), Moreover, despite an overall good sensitivity (range 81-96\%), they have relatively poor performance (sensitivity varies from $\sim 70 \%$ in MV2K to $\sim 10 \%$ in VPSPr) in atypical subtypes such as MV2K, MM2C, MM2T, and VPSPr $[1,19,50]$. Thus, clinical parameters and codon 129 genotype need to be taken into account with the diagnostic investigations' results to achieve the most accurate clinical diagnosis [62, 93]. Finally, in the absence of a post-mortem neuropathological examination, an unknown number of atypical prion cases skip recognition.

The recent development of the RT-QuIC assay provided a reliable and robust tool to improve the early diagnosis of human prion diseases [94]. Results obtained in several laboratories with the so-called first generation of this assay (also termed previous QuICCSF, or PQ-CSF), which uses a full-length (23-231) hamster recombinant prion protein as substrate, demonstrated a $73-100 \%$ sensitivity and, most significantly, a $98-100 \%$ specificity in CJD (Table 2) $[5,13$, $50,54,59,81]$.

The assay demonstrated a good reproducibility in the inter-laboratory setting and across various CSF storage conditions, such as short-term CSF storage at different temperatures, long-term storage, repeated freezing and thawing cycles $[21,55]$. The use of a chimeric version of PrP rather than wild-type hamster PrP yielded similar diagnostic accuracy in a population of both genetic and sporadic CJD, and a few FFI cases [22].

Modifications in the protocol, leading to the RT-QuIC of the second generation (also termed improved QuIC CSF, or IQ-CSF), allowed the experimental run time to be reduced to 1-2 days only and lead to a further overall increase in the sensitivity of the assay without affecting specificity [60]. These modifications included the increase in temperature during incubation, the addition of sodium dodecyl sulfate (SDS) to the reaction mix, and the substitution of full-length $\operatorname{PrP}$ with a truncated form of hamster 
Table 2 CSF RT-QulC protocols and diagnostic accuracy in prion disease

\begin{tabular}{|c|c|c|c|c|c|c|}
\hline PrP Substrate & Reaction buffer & Parameters & Cohort size $(\mathrm{N})^{\mathbf{a}}$ & Sens. & Spec. & Ref. \\
\hline \multirow[t]{9}{*}{ Hu 23-231 } & \multirow{6}{*}{$\begin{array}{l}0.06-0.1 \mathrm{~g} / \mathrm{L} \text { substrate, } \\
15 \mu \mathrm{L} \text { seed, } 500 \mathrm{mM} \mathrm{NaCl}\end{array}$} & \multirow{6}{*}{$\begin{array}{l}30 \mathrm{~s} \text { circular shaking, } \\
30 \mathrm{~s} \text { incubation, } 2 \mathrm{~min} \\
\text { break, } 37^{\circ} \mathrm{C}\end{array}$} & 18 def sCJD & $83 \%$ & & \multirow[t]{6}{*}{ [5] } \\
\hline & & & 35 def non-CJD & & $100 \%$ & \\
\hline & & & 25 prob. sCJD & $100 \%$ & & \\
\hline & & & $130 \mathrm{ctrl}$ & & $100 \%$ & \\
\hline & & & 16 prob. sCJD & $87 \%$ & & \\
\hline & & & $14 \mathrm{ctrl}$ & & $100 \%$ & \\
\hline & \multirow{3}{*}{$\begin{array}{l}0.05 \mathrm{~g} / \mathrm{L} \text { substrate, } 5 \mu \mathrm{L} \\
\text { seed, } 500 \mathrm{mM} \mathrm{NaCl}\end{array}$} & & $24 \mathrm{gCJD}$ & $83 \%$ & - & \multirow[t]{3}{*}{ [81] } \\
\hline & & & 20 GSS & $90 \%$ & - & \\
\hline & & & $12 \mathrm{FFI}$ & $83 \%$ & - & \\
\hline \multirow[t]{10}{*}{ На 23-231 } & \multirow{10}{*}{$\begin{array}{l}0.1 \mathrm{~g} / \mathrm{L} \text { substrate, } 15 \mu \mathrm{l} \\
\text { seed, } 300 \mathrm{mM} \mathrm{NaCl}\end{array}$} & \multirow{10}{*}{$\begin{array}{l}1 \text { min double orbital } \\
\text { shaking } 600 \text { rpm, } 1 \mathrm{~min} \\
\text { rest, } 42^{\circ} \mathrm{C}\end{array}$} & cohort 1: 56 def sCJD & $91 \%$ & & \multirow[t]{4}{*}{ [54] } \\
\hline & & & $52 \mathrm{ctrl}$ & & $98 \%$ & \\
\hline & & & cohort 2: 67 def sCJD & $87 \%$ & & \\
\hline & & & $52 \mathrm{ctrl}$ & & $100 \%$ & \\
\hline & & & 15/13 def/prob. sCJD & $79 \%$ & & \multirow[t]{2}{*}{ [59] } \\
\hline & & & $43 \mathrm{ctrl}$ & & $100 \%$ & \\
\hline & & & 179/97/29 def/prob./pos sCJD & $81 \%$ & & \multirow[t]{3}{*}{ [50] } \\
\hline & & & 46 gCJD & $91 \%$ & & \\
\hline & & & $348 \mathrm{ctrl}$ & & $99 \%$ & \\
\hline & & & 24/26 def/prob. sCJD & $73 \%$ & - & [13] \\
\hline \multirow[t]{3}{*}{ Ha 14-128 - Sheep 141-234 (chimera) } & \multirow{3}{*}{$\begin{array}{l}0.1 \mathrm{~g} / \mathrm{L} \text { substrate, } 15 \mu \mathrm{L} \\
\text { seed, } 170 \mathrm{mM} \mathrm{NaCl}\end{array}$} & \multirow{3}{*}{$\begin{array}{l}1 \text { min double orbital } \\
\text { shaking, } 600 \mathrm{rpm} \text {, } \\
1 \text { min rest, } 42^{\circ} \mathrm{C}\end{array}$} & 64 def sCJD & $80 \%$ & & \multirow[t]{3}{*}{ [22] } \\
\hline & & & $39 \mathrm{gCJD}$ & $100 \%$ & & \\
\hline & & & $400 \mathrm{ctrl}$ & & $99 \%$ & \\
\hline \multirow[t]{19}{*}{ Ha 90-231 } & \multirow{19}{*}{$\begin{array}{l}0.1 \mathrm{~g} / \mathrm{L} \text { substrate, } 15-30 \mu \mathrm{L} \\
\text { seed, } 300 \mathrm{mM} \mathrm{NaCl}, 0,002 \% \text { SDS }\end{array}$} & \multirow{19}{*}{$\begin{array}{l}1 \text { min double orbital } \\
\text { shaking } 700 \mathrm{rpm} \text {, } \\
1 \text { min rest, } 55^{\circ} \mathrm{C}\end{array}$} & 10/1 def/prob. sCJD & $91 \%$ & - & \multirow[t]{3}{*}[60]{} \\
\hline & & & 43/5 def/prob. sCJD & $96 \%$ & & \\
\hline & & & $39 \mathrm{ctrl}$ & & $100 \%$ & \\
\hline & & & 36/68 def/prob. sCJD & $94 \%$ & & \multirow[t]{3}{*}{ [36] } \\
\hline & & & $64 \mathrm{ctrl}$ & & $100 \%$ & \\
\hline & & & 111 sCJD & $92 \%$ & & \\
\hline & & & 15 gCJD & $93 \%$ & & \multirow[t]{3}{*}{ [30] } \\
\hline & & & $67 \mathrm{ctrl}$ & & $98.5 \%$ & \\
\hline & & & 63 sCJD, $14 \mathrm{ctrl}^{\mathrm{b}}$ & $95 \%$ & $100 \%$ & \\
\hline & & & 10/12 def/prob. sCJD & $95 \%$ & & \multirow[t]{2}{*}{ [13] } \\
\hline & & & $17 \mathrm{ctrl}$ & & $100 \%$ & \\
\hline & & & 116/73 def/prob. sCJD & $93 \%$ & & \multirow[t]{3}{*}{ [31] } \\
\hline & & & 33 gCJD & $100 \%$ & & \\
\hline & & & $100 \mathrm{ctrl}$ & & $100 \%$ & \\
\hline & & & 61/41 def/prob. sCJD & $96 \%$ & & \multirow[t]{2}{*}{ [29] } \\
\hline & & & $80 \mathrm{ctrl}$ & & $100 \%$ & \\
\hline & & & 439 def sCJD & 93 & & [72] \\
\hline & & & 31 gCJD & $97 \%$ & & \\
\hline & & & $69 \mathrm{ctrl}$ & & $98.5 \%$ & \\
\hline
\end{tabular}

Abbreviations: Hu Human, Ha Hamster, prob Probable, pos Possible, def Definite, ctrl Controls, sens. Sensitivity, spec. Specificity, sCJD Sporadic CJD, gCJD Genetic CJD, FFI Fatal familial insomnia, GSS Gerstmann-Sträussler-Scheinker disease

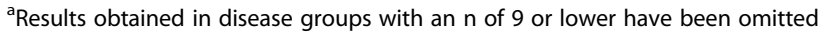

${ }^{\mathrm{b}}$ Prospective cohort 
PrP (90-231) as substrate. After the initial application of the IQ-CSF to a small cohort of samples, several groups successfully applied the new protocol to larger cohorts comprising in total about 1000 prion cases, comprehensive of the broad spectrum of prion disorders, and 500 controls [13, 29-31, 36, 72] (Table 2). Two independent studies specifically compared the first and second generation RTQuIC $[31,36]$ in the same patient population, and both of them confirmed the higher sensitivity of the IQ-CSF compared to the first generation assay (Table 2).

Regarding the application of RT-QuIC to other tissues and body fluids, recent promising studies that used either the olfactory mucosa or skin biopsies as seed showed high test sensitivities from 89 to $100 \%$, suggesting equal or even better diagnostic accuracy than CSF RT-QuIC $[29,51,59,61]$.

In summary, regardless of the type of protocol (Table 2 ), all studies conducted to date demonstrated the remarkable added diagnostic value of prion RT-QuIC compared to surrogate biomarkers. However, some methodological issues remain, limiting the full application of the assay as a screening test in RPDs. Indeed, RT-QuIC is a complicated and costly assay, which requires a continuous supply of recombinant protein, which is often challenging to recruit for laboratories that do not produce the substrate in-house. Moreover, the second generation RT-QuIC still needs validation in the inter-laboratory setting.

Finally, questions about the assay's presumed absolute specificity and the effect of prion strains and disease subtypes on test sensitivity deserve further discussion.

\section{Current unsolved questions and potential limitations Is prion $R T$-QulC fully specific?}

The development of an RT-QuIC assay with sufficient sensitivity requires a high concentration of recombinant substrate and a reaction environment favoring the seeded aggregation mechanism. Consequently, there is a need to monitor any spontaneous substrate aggregation that may generate false-positive signals. To this aim, samples are loaded four times in the standard RT-QuIC set-up, and a definite positive read requires that at least two of the four replicates generate a signal above a pre-set threshold, whereas a single positive well is considered an unclear result, prompting the repetition of the assay.

Some studies on prion RT-QuIC reported a few unexpected positive results [22, 30, 50, 54]. However, most of them involved patients who did not receive an autopsy confirmation, leaving open the possibility that they were clinically unrecognized atypical prion cases. Indeed, a retrospective analysis of such cases favors this interpretation. As an exception, Foutz and co-workers [30] reported a positive RT-QuIC in a patient with the neuropathological diagnosis of dementia with Lewy bodies (DLB). In order to investigate this sample deeply, the researchers precipitated the brain homogenate in phosphotungstic acid, a commonly used compound that allows the enrichment of pathogenic prions. Western blot analysis of the pellet revealed low but above-threshold levels of PK-resistant $\mathrm{PrP}^{\mathrm{Sc}}$, which led to the conclusion that this might have been a case of subclinical prion disease, who died from DLB comorbidity. In other recent reports [38, 72], an autopsy-verified case of steroidresponsive encephalopathy with status epilepticus and one with dementia of mixed type ( $\mathrm{AD}$ and vascular) also received a false-positive result, respectively, by $\mathrm{PQ}$ and IQ-CSF RT-QuIC. Thus, although false readouts by prion RT-QuIC seem to be extremely rare and some of them may even represent real prion cases, the test does not seem to provide yet a level of diagnostic certainty comparable to a neuropathological examination. Consequently, currently updated diagnostic criteria still require a post-mortem examination for the definite diagnosis of sporadic or acquired prion disease [94].

\section{To what extent does the disease subtype affect the sensitivity of the assay?}

Only a few studies examined a significant number of CJD stratified according to the disease subtype. Using the first generation of the assay, Cramm et al. [22] reported that neither the $\operatorname{PrP}^{\mathrm{Sc}}$ type nor the codon 129 genotype alone had a significant impact on the RT-QuIC response. Nevertheless, $\operatorname{PrP}^{\mathrm{Sc}}$ type 1 patients carrying $\mathrm{MM}$ at codon 129 showed a faster response that those carrying MV or VV (who most commonly harbor $\mathrm{PrP}^{\mathrm{Sc}}$ type 2). Moreover, disease duration, which, once again, mainly depends on the disease subtype, had an impact on the test results, with patients with a shorter disease course (who most commonly have $\operatorname{PrP}^{\mathrm{Sc}}$ type 1 ) showing higher seeding efficiency in the RT-QuIC response. In a large study including 186 pathologically confirmed sCJD cases with subtype classification, [50] RT-QuIC sensitivity varied according to the codon 129 genotype and was higher in MM (84.2\%) than in MV (72.2\%) or VV (79.5\%) cases. Besides, there was convincing evidence that the assay sensitivity was significantly lower in the atypical subtypes linked to $\operatorname{PrP}^{\mathrm{Sc}}$ type 2 (i.e., VV2, MV2K, MM2T, and MM2C).

As far as the second generation RT-QuIC is concerned, Foutz et al. [30] found that RT-QuIC positivity was significantly associated with older age, a shorter disease course, and a higher frequency of ataxia, features that are all linked to the two most common subtypes, MM1 and VV2. Furthermore, RT-QuIC was more frequently negative in SCJD MM2. However, there were no correlations between the RT-QuIC result and codon 129 
polymorphism alone, gender, electroencephalography, or MRI findings. Franceschini et al., [31] in a large cohort of cases with disease subtype classification, found comparable levels of sensitivities in the three most common subtypes, namely $\mathrm{MM}(\mathrm{V}) 1, \mathrm{VV} 2$ and $\mathrm{MV} 2 \mathrm{~K}$, but confirmed the findings of reduced sensitivity of IQ CSF RTQuIC in both MM2 subtypes (i.e., MM2C and MM2T or sFI). Similarly, Rhoads and co-workers recently reported that atypical sporadic prion disease subtypes (i.e., sFI, VPSPr, sCJD VV1, and sCJD MM2C) are more likely to have false negative RT-QuIC results [72].

As for the SCJD subtypes, only a few studies tested a significant number of cases affected by genetic prion disease. Using PQ RT-QuIC, Sano et al. [81] analyzed a small cohort of CSF specimens from patients with genetic CJD (E200K, $n=22$; V203I, $n=2$ ), GSS (P102L, $n=20)$ or FFI (D178N, $n=12$ ). Results were comparable between the groups, with only a slightly higher sensitivity in GSS compared to FFI and gCJD (90\% vs. 83.3\%). In contrast, other groups, using different protocols, consistently reported a reduced sensitivity of RT-QuIC in FFI (0-57\%), and GSS (0-50\%) compared with gCJD (83.3-100\%) [12, 22, 30, 31, 72]. Moreover, RT-QuIC gave positive results in one asymptomatic E200K carrier, who did not develop the disease after a 1-year follow-up [88]. Finally, based on the few results obtained in GSS and CJD cases carrying rare PRNP mutations, it is very likely that different disease-associated mutations might influence the test's diagnostic value [31]. The outstanding performance of prion RT-QuIC, and the discovery that other proteins involved in neurodegenerative diseases show a seeding-conversion behavior similar to that of PrP, prompted the scientific community to develop an RT-QuIC assay that could detect protein amyloids in more common neurodegenerative diseases such as Alzheimer's disease (AD) and Parkinson's Disease (PD) in the CSF of diseased individuals.
In the next sections, we will review the successful adaptation of the RT-QuIC for the detection of pathogenic forms of $\alpha$-syn and, to a lesser extent, of tau in human CSF.

\section{Diagnostic value of a-syn RT-QuIC in synucleinopathies}

Synucleinopathies, including PD, DLB, and multiple system atrophy (MSA) are neurodegenerative diseases characterized by the intracellular accumulation of toxic $\alpha$ syn aggregates [87] forming Lewy bodies (LB) and neurites in PD and DLB and oligodendroglial cytoplasmic bodies, and much rarer neuronal inclusions in MSA [24]. Similar to prion diseases, synucleinopathies manifest an extensive phenotypic heterogeneity involving clinical presentation, response to therapy, and rate of progression (Table 3). Moreover, the recognition that two other rarer syndromes, namely pure autonomic failure (PAF) and isolated rapid eye movement (REM) sleep behavior disorder (iRBD), characterized by the early $\alpha$ syn accumulation in the peripheral autonomic system and lower brainstem, represent prodromal syndromes of both MSA and LB-related disorders (LBD), has widened the clinical spectrum of synucleinopathies and further complicated the early clinical diagnosis of these disorders $[40,46]$. Increasing evidence demonstrating structural differences in the $\alpha$-syn aggregates between MSA and LBD suggests that different conformational strains of $\alpha$-syn may contribute to their clinicopathological heterogeneity [41]. Nowadays, even the early in vivo diagnosis of a full-blown LBD can be tricky since it requires the distinction from both atypical parkinsonisms such as progressive supranuclear palsy (PSP) and corticobasal degeneration (CBD), which are primary tauopathies, and other neurodegenerative dementias such as AD or frontotemporal dementia (FTD). Despite the progressive improvement of the accuracy of diagnostic criteria for the identification of synucleinopathies in vivo, the clinicopathological rate of accordance in autopsy verified

Table 3 The spectrum of synucleinopathies: Main clinical features and diagnostic tests

\begin{tabular}{|c|c|c|c|c|}
\hline Disease & Mean duration & Main affected brain regions & Main clinical features & Main diagnostic tests \\
\hline$\overline{\mathrm{PD}}$ & $10-20$ & Brainstem & L-DOPA responsive parkinsonism & $\overline{\text { DaTSCAN (ioflupane) }}$ \\
\hline DLB & 8 & Neocortices, limbic system, brainstem & $\begin{array}{l}\text { Cognitive decline with fluctuations, } \\
\text { parkinsonism, visual hallucinations, } \\
\text { changes to cognitive ability, RBD }\end{array}$ & $\begin{array}{l}\text { DaTSCAN (ioflupane), } \\
\text { MIBG, PSG, EEG }\end{array}$ \\
\hline MSA & $7-9$ & Striatum, cerebellum, brainstem & $\begin{array}{l}\text { Autonomic failure with various degree } \\
\text { of parkinsonism, cerebellar and } \\
\text { pyramidal signs }\end{array}$ & $\begin{array}{l}\text { DaTSCAN (ioflupane), } \\
\text { MRI, autonomic tests, } \\
\text { FDG-PET }\end{array}$ \\
\hline PAF & a & PNS & Isolate autonomic failure & Autonomic tests \\
\hline iRBD & $a$ & Lower brainstem & IRBD & PSG \\
\hline
\end{tabular}

Abbreviations: PD Parkinson's disease, DLB Dementia with Lewy bodies, MSA Multiple system atrophy, PAF Pure autonomic failure, iRBD Isolate REM sleep behavior disorder, PNS Peripheral nervous system, MIBG Metaiodobenzylguanidine, PSG Polysomnography, EEG Electroencephalography, FDG-PET Fluorodeoxyglucosepositron emission tomography

a Duration as "isolate" disorder, and rate of phenotypic conversion are highly variable 
cohorts is often less than optimal (i.e., 92.6\% for PD, 81.6\% for DLB, $62-78.8 \%$ for MSA) [48, 58, 67, 73].

Moreover, current diagnostic criteria usually require the association of clinical findings, multiple diagnostic investigations, and an adequate follow-up of several years, to reach the accurate identification of the disorder [34, 66]. Most significantly, imaging, neurophysiological and neuropsychological tests are only supportive of the clinical findings, since they are not specific markers of the pathologic process (i.e., the accumulation of abnormal $\alpha$-syn aggregates). Thus, in the era in which both clinical and scientific interests focus on the early recognition of neurodegenerative diseases, post-mortem neuropathology remains the gold standard for the definite diagnosis of synucleinopathies given the lack of an in vivo specific molecular marker.

Increasing evidence supporting the prion-like behavior of $\alpha$-syn, together with those indicating that the seeding conversion mechanism could be replicated in vitro by aggregation assays such as PMCA [43, 82], prompted researchers to adapt the RT-QuIC assay for the detection of pathogenic $\alpha$-syn. The key assay parameters and the main results obtained in the studies completed to date are shown in Table 4.

The first successful application of $\alpha$-syn RT-QuIC [27] involved a limited cohort of CSF derived from clinically diagnosed PD $(n=20)$ and neuropathologically confirmed DLB $(n=12)$ cases. Results showed a $92-95 \%$ sensitivity and a $100 \%$ specificity against 35 healthy controls and 5 $\mathrm{PSP} / \mathrm{CBD}$ cases. Interestingly, three samples derived from patients affected by iRBD also gave a positive result, providing preliminary evidence that, in the clinical frame of a prodromic syndrome, the RT-QuIC may predict the future onset of a synucleinopathy. In an adaptation of the PMCA technique for the detection of misfolded $\alpha$-syn that firmly reminds the RT-QuIC, as it makes use of a recombinant substrate, intermittent shaking and ThT, Shahnawaz et al. [83] measured $\alpha$-syn seeding activity in PD patients $(n=76)$ and controls with other neurological diseases $(n=97)$. This novel $\alpha$-syn PMCA assay distinguished PD from other neurologic disorders with $88.5 \%$ sensitivity and $97 \%$ specificity (Table 4 ). The research group also found that $\alpha$-syn PMCA values in PD significantly correlated with disease severity according to the scores on the Hoehn and Yahr scale.

Whereas the original assay [27] showed an average lag phase of $50-60 \mathrm{~h}$ and required an observation time of $120 \mathrm{~h}$, modifications of the experimental setup [37], including the use of a novel recombinant protein carrying the K23Q mutation, allowed the reduction of the lag phase to approximately $20 \mathrm{~h}$ and the total assay duration to $48 \mathrm{~h}$. Most significantly, sensitivity remained above $90 \%$ and specificity $100 \%$ for both PD $(n=12)$ and DLB $(n=17)$ against $\mathrm{AD}$ cases $(n=16)$ and controls $(n=12)$.
To further explore its diagnostic value in the clinical contest, the authors applied $\alpha$-syn RT-QuIC to CSF samples from a prospective observational cohort of 118 patients with parkinsonism of uncertain clinical diagnosis at lumbar puncture (LP) [89]. The overall diagnostic accuracy in distinguishing $\alpha$-synucleinopathies from other disorders and controls was $84 \%$ (sensitivity $=75 \%$, specificity $=94 \%$ ), with a significant decrease in accuracy in comparison to the two previous studies, which analyzed relatively small groups of typical and, for the most part, neuropathologically confirmed LBD cases. Intriguingly, sensitivity significantly correlated with the type of synucleinopathy, being $100 \%$ in DLB, $84 \%$ in PD, and only $35 \%$ in MSA.

In another study, the RT-QuIC was used to detect $\alpha$ syn seeding activity in patients carrying mutations in LRRK2 (Leucine-rich repeat kinase 2 gene) [32], the most common cause of genetic PD. In a blind analysis that compared these carriers (LRRK2-PD, $n=15$; asymptomatic carriers, $n=16$ ) with either idiopathic PD or healthy controls, the assay sensitivity was surprisingly rather low (40\% in LRRK2-PD, 19\% in the asymptomatic carriers) in the former group, whereas it was comparable to previous studies (90\%) in the idiopathic (non-genetic) PD group. The assay specificity, however, dropped to $80 \%$. The finding supports the prevalent idea that LRRK2 carriers may not necessarily have an underlying synucleinopathy. Indeed, neuropathological studies demonstrated, at least in a few of such cases, a nigral degeneration in the absence of protein deposition [53] or the presence of aggregates made up by misfolded tau [39].

The high sensitivity of $\alpha$-syn RT-QuIC for the diagnosis of DLB was confirmed in another study evaluating a series of atypical cases referred to laboratories specialized in CJD diagnosis because of a rapidly progressive course [12]. The diagnostic accuracy, calculated on the cases assessed neuropathologically $(n=77)$, irrespectively of the clinical diagnosis (i.e., only 8 out of 27 LBD cases had a clinical diagnosis of probable DLB) resulted in a $92.8 \%$ sensitivity and $95.9 \%$ specificity. Additional results obtained in a clinical cohort of 36 cases showed an $85 \%$ sensitivity in the group of probable DLB and, intriguingly, a $0 \%$ sensitivity in those $(n=6)$ with possible DLB. Finally, there were no positive reads also in a group of 10 cases with a clinical diagnosis of probable AD.

In the first attempt to cross-validate $\alpha$-syn seeding aggregation assays developed by independent laboratories, Kang et al. [44] undertook a blinded study in which the two participating groups (Soto's lab and Green's lab) analyzed CSF from the same subjects in the BioFIND cohort [45], including 105 clinically typical and well-defined moderate PD patients and 79 healthy controls. Despite the different denominations 
Table 4 CSF RT-QulC protocols and diagnostic accuracy in synucleinopathies

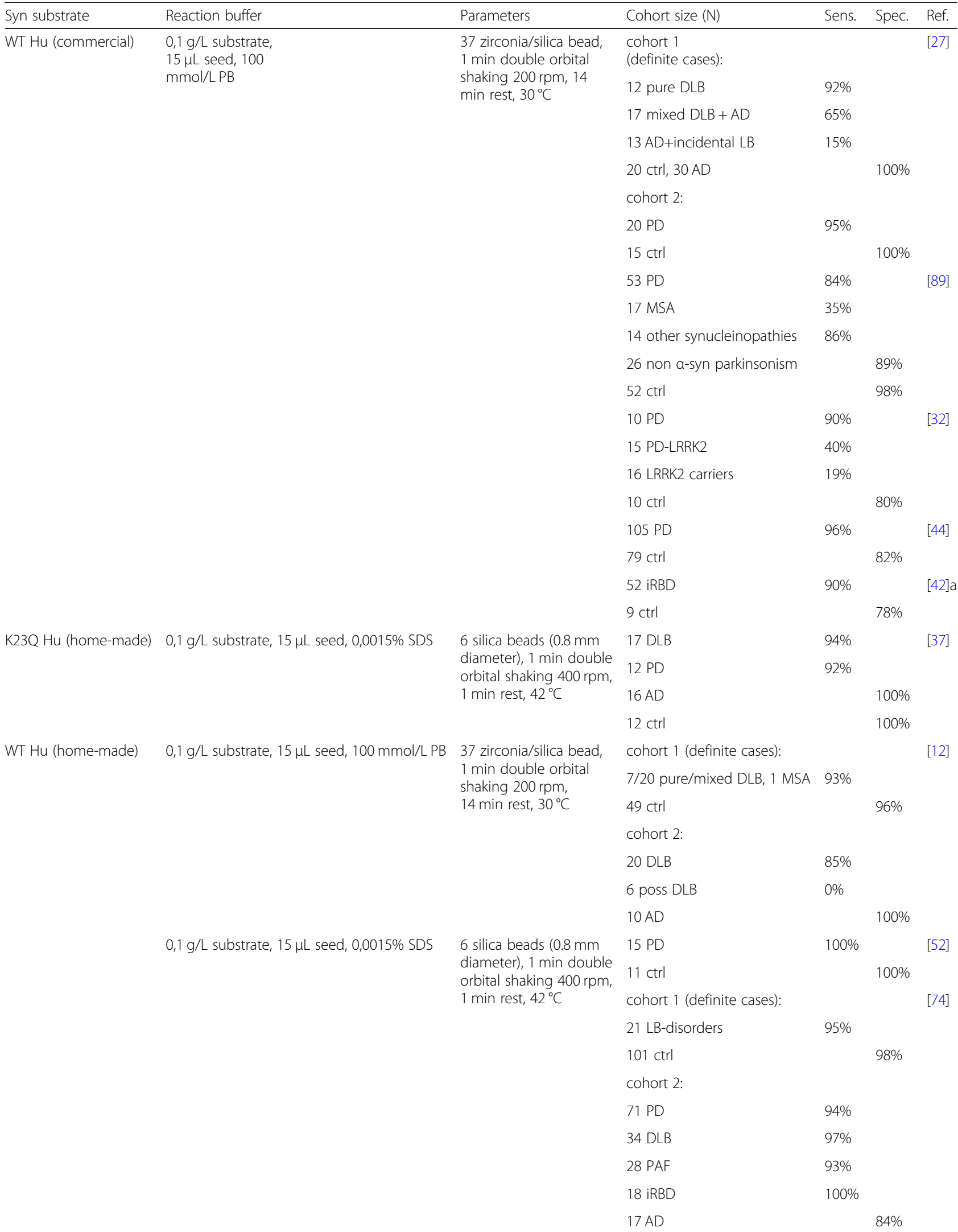


Table 4 CSF RT-QulC protocols and diagnostic accuracy in synucleinopathies (Continued)

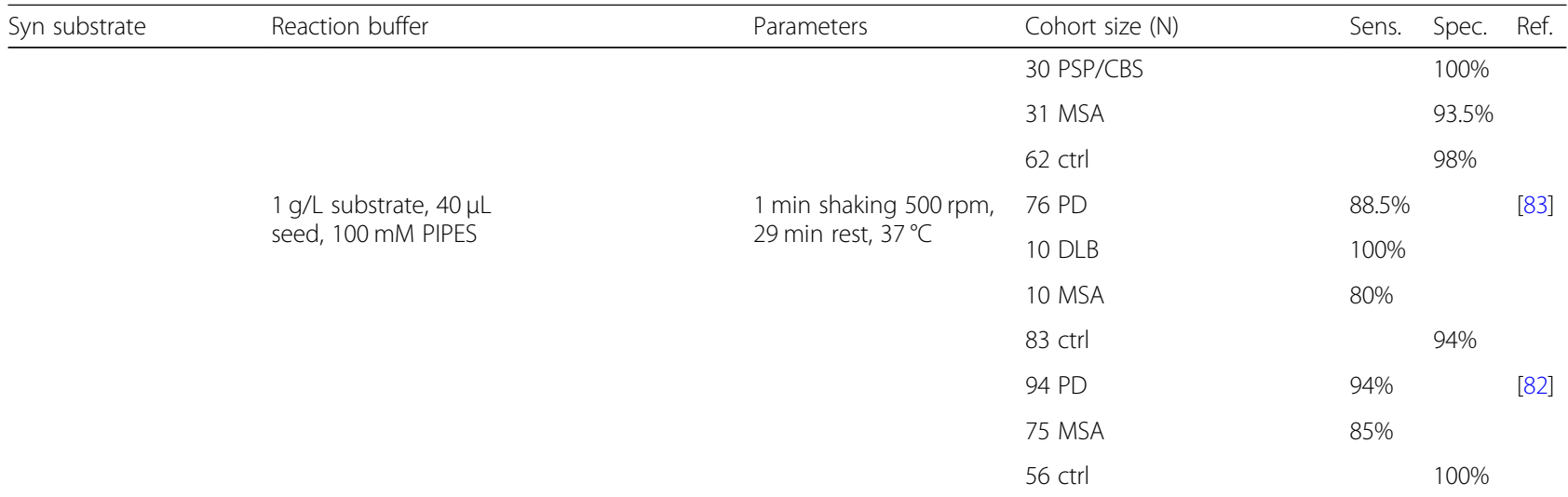

Diagnostic groups/cohorts are meant to be clinically defined as probable cases, unless otherwise specified. Sensitivities values for diagnostic groups $\leq 5$ are not included

Abbreviations: Hu Human, WT Wild type, LB Lewy bodies, DLB Dementia with LB, AD Alzheimer's disease, PD Parkinson's disease, MSA Multiple system atrophy, $i R B D$ Isolate REM sleep behavior disorder, PSP Progressive supranuclear palsy, CBS Corticobasal syndrome, PAF Pure autonomic failure, prob Probable, poss Possible, ctrl Controls

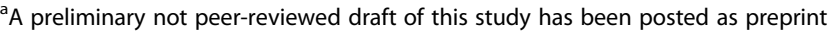

(RT-QuIC and PMCA), the two assays did not differ significantly in the basic principles. Overall, $92 \%$ of subjects showed concordant results across both assays. Eleven subjects were positive by RT-QuIC but negative by PMCA, and four subjects were positive by PMCA but negative by RT-QuIC. Sensitivity and specificity for the PMCA and RT-QuIC assays were $95.2 \% / 89.9 \%$, and $96.2 \% / 82.3 \%$, respectively. Clinical characteristics of false-positive and -negative subjects were not different from true-negative and -positive subjects, respectively.

In a recent study, using the standard protocol developed by Caughey's lab, but a wild-type recombinant $\alpha$ synuclein substrate rather than the mutant form used by Groveman et al. [37], Rossi and colleagues [74] applied the RT-QuIC to the largest cohort of CSF samples examined to date. They included 439 clinically wellcharacterized, or post-mortem verified, patients with parkinsonism or dementia, and, for the first time, a significant number of patients with iRBD and PAF. The RT-QuIC assay detected $\alpha$-syn seeding activity across the spectrum of LBD, including DLB, PD, iRBD, and PAF, with an overall sensitivity of $95.3 \%$. Surprisingly, all but two patients with MSA showed no $\alpha$-syn seeding activity in the applied experimental setting. Finally, the assay demonstrated $98 \%$ specificity in a neuropathological cohort of 101 cases lacking LB pathology. In a recent study [82], a modified $\alpha$-syn PMCA discriminated between PD and MSA pathologies based on the fluorescent signal profile in a cohort of 94 samples of CSF from patients with PD, 75 from patients with MSA and 56 from control individuals with other neurological diseases. The overall sensitivity for the identification of PD and MSA vs. controls was 93.6 and $84.6 \%$, respectively, and, in both cases, specificity was $100 \%$ (Table 4).
Furthermore, PD was correctly distinguished from MSA in 146 of the 153 samples analyzed, resulting in an overall sensitivity of $95.4 \%$.

In the most recent of these studies, Iranzo and colleagues [42] applied the RT-QuIC developed by Fairfoul et al. [27] in 52 polysomnographically-confirmed iRBD patients, and only nine, matched, healthy controls. The assay gave a positive result in $47(90.4 \%)$ patients and two (22.2\%) controls. During follow-up, $32(61.5 \%)$ patients received a diagnosis of PD or DLB $3.4 \pm 2.6$ years after LP, and 31 (96.9\%) of these were positive for CSF $\alpha$-syn RT-QuIC, while none of the controls developed a synucleinopathy.

Lastly, following the successful application of PrP RTQuIC on peripheral tissues, De Luca et al., [23] applied the RT-QuIC to detect misfolded forms of $\alpha$-syn in brushes of olfactory mucosa. However, in contrast to the high accuracy in prion disease, $\alpha$-syn RT-QuIC showed a suboptimal performance $(65.5 \%$ sensitivity, and $84.4 \%$ specificity). Notably, a few PSP samples gave a definite read, suggesting a certain degree of cross-reactivity with misfolded tau.

\section{Current limitations and future perspectives}

The results of the studies mentioned above demonstrated that RT-QuIC is highly accurate in diagnosing synucleinopathies, with values almost as good as those previously obtained in prion disease. However, they also showed divergent findings and significant variability in the predictive values of the test, which are likely related to critical variables such as the choice of recombinant substrate and experimental setting, the accuracy of the final diagnosis (i.e., neuropathological vs. clinical), and the number of examined cases in each diagnostic group. 
For example, specificity was very high among studies focusing on neuropathologically verified cohorts (95.9-100\%) [12, 27, 37, 74], with two of them reporting a full-specificity [27, 37]. In these cohorts, false-positive readouts involved only four cases, two with $\mathrm{AD}$, one with Wernicke's encephalopathy, and one with unspecified encephalitis [12, 74]. However, whereas the two cases reported by Rossi et al. [74] showed a consistent positivity in repeated analyses, the other two [12] gave an opposite negative result when re-analyzed after breaking the blinded code. Thus, while the latter two cases suggest a true falsepositivity due to the self-aggregation of the recombinant $\alpha$-syn during the RT-QuIC reaction, the consistent positive signal in the former two samples is also compatible with a real $\alpha$-syn seeding activity. Indeed, the presence of LB pathology could not be rule out in these cases, given that the histopathological examination did not include the olfactory bulb [2, 71], or the peripheral autonomic ganglia [16, 33], which can be first sites of $\alpha$-syn accumulation.

In contrast to neuropathologically verified cohorts, the results obtained in clinical groups consistently showed false-positive reads $[12,32,42,44,74,89]$, resulting in a drop of specificity. The incomplete specificity was particularly evident with the assay developed by Green's lab, which is also the most frequently applied to date. Specificity was $100 \%$ in the original study [27], but then dropped to $94 \%$ [89], $82 \%$ [44], and even $78 \%$ in the latter [42], which, however, included only nine controls.

In some cases, the percentage of apparently falsepositive results in clinically diagnosed cohorts was related to a specific pathological condition, leaving different open interpretations. For example, in AD, LBrelated co-pathology is a relatively frequent finding at post-mortem examination. Accordingly, the 7 out of 43 AD cases that were positive by RT-QuIC in the study by Rossi et al. [74] might have developed LBs as significant co-pathology, indicating a real positivity rather than a false positive readout.

Similarly, the evaluation of unexpected positive results in clinical "healthy" controls should take into account that Lewy pathology affects $5-8 \%$ of people over 60 in the absence of extrapyramidal signs $[25,28]$. Since parkinsonian signs appear when about $30 \%$ of dopaminergic neurons of substantia nigra are lost [35], these subjects may represent prodromal-PD. The observed $100 \%$ specificity in the presenile control group of subjects examined by Rossi et al. [74] is consistent with this conclusion and seems to exclude spontaneous aggregation as the leading cause of false-positive results in their assay.

Concerning the diagnostic sensitivity, most studies demonstrated high values (above 90\%) across the entire spectrum of syndromes associated with LB synuclein pathology $[12,27,37,52,74,89]$. In a more in-depth analysis of the false-negative cases, clinical features did not show any specific association in any of the studies, suggesting that the negative response might be more likely due to the sample matrix composition rather than the underlying pathology.

The variable but generally very low sensitivity of $\alpha$-syn RT-QuIC in MSA deserves a separate comment. Two studies to date analyzed a significant number of MSA cases. They reported a 6.1\% [74] and 35\% [89] overall sensitivity, indicating that $\alpha$-syn RT-QuIC, in the currently proposed set-ups, consistently fails to reveal $\alpha$-syn pathogenic species in the CSF of MSA patients. Thus, a negative rather than a positive result might be applied to support MSA's clinical diagnosis. The striking difference in seeding activity in comparison to LBD specimens may rely on a different conformation of the pathogenic seed, referred to as strain, as suggested by recent in vitro and ex vivo studies [82].

Nonetheless, when compared to controls, CSF samples from MSA patients gave a higher number of positive reads than controls, suggesting a certain degree of crossreactivity with the $\alpha$-syn recombinant substrate. Thus, the use of novel substrates or the modification of other parameters in the $\mathrm{RT}-\mathrm{QuIC}$ reaction might allow to increase the sensitivity for $\alpha$-syn seeding activity in MSA cases and to distinguish them from controls and other synucleinopathies.

\section{CSF 4R-tau RT-QuIC: a potential diagnostic aid in the differential diagnosis of parkinsonism and cognitive decline?}

Tau RT-QuIC represents the latest developed adaptation of the seeding conversion assays. Tau is a protein whose insoluble filaments are found in AD as well as in several other neurodegenerative diseases, collectively termed tauopathies. In vitro evidence $[26,80]$ indicated that tau possesses self-seeding properties resembling those of PrP. Therefore, following the success obtained in prion diseases and synucleinopathies, researchers also attempted to adapt the RT-QuIC for the detection of misfolded tau in the CSF.

However, the tau protein complexity arising from the presence of six isoforms, which differ one another for the inclusion of amino-terminal regions $(0 \mathrm{~N}, 1 \mathrm{~N}$, and 2 $\mathrm{N}$, respectively) and either 3 or 4 microtubule-binding repeats ( $3 R$ and $4 R$ tau, respectively), challenged the development of tau RT-QuIC until very recently.

The first successful RT-QuIC assay for the detection of misfolded tau species in the CSF was put forward by Saijo and co-workers [78]. The assay was initially tuned to accurately detect Pick's Disease (PiD), a tauopathy in which aggregates exclusively comprise the $3 \mathrm{R}$ tau isoform. However, the test only worked with brain tissue or 
post-mortem CSF as seeds, limiting its potential application in vivo.

Using similar RT-QuIC conditions, but employing different tau constructs, the same group adapted the RTQuIC for the detection of tau misfolded species from mixed (3R/4R) tauopathies, such as AD and chronic traumatic encephalopathy patients, or $4 \mathrm{R}$ tauopathies, such as PSP and CBD [49, 79]. While the former assay, once again, only worked with a brain homogenate as seed, the $4 \mathrm{R}$-specific construct also revealed promising results in the samples collected in vivo, although the accuracy was significantly lower in the CSF samples obtained in vivo compared to those collected post-mortem [49, 57, 78, 79]. In summary, tau RT-QuIC embodies enormous potential but needs to be refined and tested in a large cohort of patients before being proposed as a valuable diagnostic tool.

\section{Conclusions}

After its original implementation 10 years ago [91], the RT-QuIC assay has gained significant ground among the available laboratory aids for the clinical diagnosis of neurodegenerative disorders. Although the assay does not distinguish between disease subtypes and its prognostic value is still far from wellestablished, RT-QuIC has become the most potent tool for the in vivo diagnosis of prion disease and has significantly impacted both clinical diagnostic criteria and clinical practice. Moreover, recent $\alpha$-syn RTQuIC set-ups provided a robust biomarker for LBrelated disorders, supporting its introduction in research diagnostic criteria and clinical trials. Finally, promising preliminary results also involve misfolded tau and the primary $3 R$ and $4 R$ tauopathies.

Despite these significant advances, however, many challenges and unanswered questions remain. The recombinant substrate, which is critical for the RTQuIC method's accuracy, must be standardized and produced on a larger scale to be more readily available to laboratories worldwide. Most recent RT-QuIC protocols need validation in the inter-laboratory setting and the accuracy of the RT-QuIC using peripheral tissues such as the skin and nasal brushes further testing. In prion disease, there is also a need for improved RT-QuIC protocols to differentiate between sCJD subtypes and detect atypical prion diseases with higher sensitivity in vivo.

In synucleinopathies, future studies should establish the impact of RT-QuIC on clinical diagnostic criteria, determine the assay performance on other accessible specimens, and search for variations in the RT-QuIC set-up that might correctly detect $\alpha$-syn seeding activity in MSA. Moreover, the application of RT-QuIC to genetically determined PD cases and other rare atypical forms of PD should help to establish the real spectrum of LB-related PD, and distinguishing PD associated with LB pathology from PD cases caused by primary neuronal degeneration in the substantia nigra without LB formation. Finally, protocols must be developed further to bring tau RT-QuIC to the level of diagnostic accuracy shown by the prion and $\alpha$-syn assays. If successful, these and other advancements will likely make the RT-QuIC of critical importance for the early diagnosis and treatment of most of these devastating disorders.

\section{Abbreviations}

RT-QulC: Real-time quaking-induced conversion assay; PrP: Prion protein; $\operatorname{PrP}^{C}$ : Cellular prion protein; $\operatorname{PrP}^{\mathrm{SC}}$ : Scrapie prion protein; rPrPsen: Hamster recombinant prion protein; CJD: Creutzfeldt-Jakob disease; SCJD: Sporadic Creutzfeldt-Jakob disease; LBD: Lewy body disease; AD: Alzheimer's disease; PK: Proteinase K; PMCA: Protein misfolding cyclic amplification; QulC: Quaking-induced conversion; ThT: Thioflavin-T; a-syn: a-synuclein; GSS: Gerstmann-Sträussler-Scheinker disease; Fl: Fatal insomnia; VPSPr: Variably protease-sensitive prionopathy; M: Methionine; V: Valine; RPDs: Rapidly progressive dementias; DW-MRI: Diffusion-weighted magnetic resonance imaging; IQ-CSF: Improved RT-QuIC CSF; SDS: Sodium dodecyl sulfate; PD: Parkinson's disease; DLB: Dementia with Lewy bodies; MSA: Multiple system atrophy; LB: Lewy bodies; PAF: Pure autonomic failure; IRBD: Isolate REM (rapid eye movement) sleep behavior disorder; PSP: Progressive supranuclear palsy; CBD: Corticobasal degeneration; FTD: Frontotemporal dementia; PNS: Peripheral nervous system; MIBG: Metaiodobenzylguanidine; PSG: Polysomnography;

EEG: Electroencephalography; MRI: Magnetic resonance imaging; FDGPET: Fluorodeoxyglucose-positron emission tomography; LP: Lumbar puncture; LRRK2: Leucine-rich repeat 2 gene; PiD: Pick's Disease

\section{Acknowledgements}

Not applicable.

\section{Authors' contributions}

NC, SB, PP designed the study; NC, PP, SB, AF wrote the main text (first draft): NC, SB produced the tables; SB produced the figures; PP critically revised the whole manuscript. All authors read and approved the final manuscript.

\section{Funding}

Ministero della Sanità, "Ricerca Corrente".

Availability of data and materials

Not applicable.

Ethics approval and consent to participate

Not applicable.

Consent for publication

Not applicable.

Competing interests

The authors declare that they have no competing interests.

\section{Author details}

'Department of Experimental, Diagnostic and Specialty Medicine (DIMES), University of Bologna, Bologna, Italy. ${ }^{2}$ RCCS, Istituto delle Scienze Neurologiche di Bologna, Ospedale Bellaria, Via Altura 1/8, 40139 Bologna, Italy. ${ }^{3}$ Department of Biomedical and Neuromotor Sciences, University of Bologna, Bologna, Italy. 
Received: 7 June 2020 Accepted: 6 July 2020

Published online: 25 July 2020

\section{References}

1. Abu-Rumeileh S, Baiardi S, Polischi B, Mammana A, Franceschini A, Green A et al (2019) Diagnostic value of surrogate CSF biomarkers for CreutzfeldtJakob disease in the era of RT-QulC. J Neurol 266(12):3136-3143

2. Adler CH, Beach TG, Zhang N, Shill HA, Driver-Dunckley E, Caviness JN et al (2019) Unified staging system for Lewy body disorders: clinicopathologic correlations and comparison to Braak staging. J Neuropathol Exp Neurol 78(10):891-899

3. Ashton NJ, Hye A, Rajkumar AP, Leuzy A, Snowden S, Suárez-Calvet M et al (2020) An update on blood-based biomarkers for non-Alzheimer neurodegenerative disorders. Nat Rev Neurol 16(5):265-284. https://doi.org/ 10.1038/s41582-020-0348-0

4. Atarashi R, Moore RA, Sim VL, Hughson AG, Dorward DW, Onwubiko HA et al (2007) Ultrasensitive detection of scrapie prion protein using seeded conversion of recombinant prion protein. Nat Methods 4(8): 645-650

5. Atarashi R, Satoh K, Sano K, Fuse T, Yamagichi N, Ishibashi D et al (2011) Ultrasensitive human prion detection in cerebrospinal fluid by real-time quaking-induced conversion. Nat Methods 17(2):175-178

6. Baiardi S, Capellari S, Bartoletti Stella A, Parchi P (2018) Unusual clinical presentations challenging the early clinical diagnosis of Creutzfeldt-Jakob disease. J Alzheimers Dis 64(4):1051-1065

7. Baiardi S, Rossi M, Capellari S, Parchi P (2019) Recent advances in the histo-molecular pathology of human prion disease. Brain Pathol 29(2): 278-300

8. Baldwin MA, Pan K, Nguyen J, Huang Z, Groth D, Serban A et al (1994) Spectroscopic characterization of conformational differences between PrPC and PrPSc: an a-helix to $\beta$-sheet transition. Philos Trans R Soc Lond Ser B Biol Sci 343(1306):435-441

9. Barria MA, Mukherjee A, Gonzalez-Romero D, Morales R, Soto C (2009) De novo generation of infectious prions in vitro produces a new disease phenotype. PLoS Pathog 5:e1000421

10. Biancalana M, Koide S (2010) Molecular mechanism of Thioflavin-T binding to amyloid fibrils. Biochem Biophy Acta 1804(7):1405-1412

11. Bizzi A, Pascuzzo R, Blevins J, Grisoli M, Lodi R, Moscatelli MEM et al (2020) Evaluation of a new criterion for detecting prion disease with diffusion magnetic resonance imaging. JAMA Neurol:e201319. https://doi.org/10. 1001/jamaneurol.2020.1319

12. Bongianni M, Ladogana A, Capaldi S, Klotz S, Baiardi S, Cagnin A et al (2019) a-Synuclein RT-QulC assay in cerebrospinal fluid of patients with dementia with Lewy bodies. Ann Clin Transl Neurol 6(10):2120-2126

13. Bongianni M, Orrù C, Groveman BR, Sacchetto L, Fiorini M, Tonoli G et al (2017) Diagnosis of human prion disease using real-time quaking-induced conversion testing of olfactory mucosa and cerebrospinal fluid samples. JAMA Neurol 74(2):55-162

14. Carswell C, Thompson A, Lukic A, Stevens J, Rudge P, Mead S et al (2012) MRI findings are often missed in the diagnosis of Creutzfeldt-Jakob disease. BMC Neurol 12:153

15. Caughey BW, Dong A, Bhat KS, Ernst D, Hayes SF, Caughey WS (1991) Secondary structure analysis of the scrapie-associated protein PrP 27-30 in water by infrared spectroscopy. Biochemistry 30(31):7672-7680

16. Cersosimo MG, Benarroch EE (2012) Autonomic involvement in Parkinson's disease: pathology, pathophysiology, clinical features and possible peripheral biomarkers. J Neurol Sci 313(1-2):57-63

17. Chiti F, Dobson CM (2017) Protein misfolding, amyloid formation, and human disease: a summary of progress over the last decade. Annu Rev Biochem 86:27-68

18. Colby DW, Zhang Q, Wang S, Groth D, Legname G, Riesner D et al (2007) Prion detection by an amyloid seeding assay. Proc Natl Acad Sci U S A 104(52):20914-20919

19. Collins SJ, Sanchez-Juan P, Masters CL, Klug GM, van Duijn C, Poleggi A et al (2006) Determinants of diagnostic investigation sensitivities across the clinical spectrum of sporadic Creutzfeldt-Jakob disease. Brain 129(Pt. 9): 2278-2287

20. Cosseddu GM, Nonno R, Vaccari G, Bucalossi C, Fernandez-Borges N, Di Bari MA et al (2011) Ultra-efficient PrP (SC) amplification highlights potentialities and pitfalls of PMCA technology. PLoS Pathog 7:e1002370
21. Cramm M, Schmitz M, Karch A, Mitrova E, Kuhn F, Schroeder B et al (2016) Stability and reproducibility underscore utility of RT-QulC for diagnosis of Creutzfeldt-Jakob disease. Mol Neurobiol 53(3):1896-1904

22. Cramm M, Schmitz M, Karch A, Zafar S, Varges D, Mitrova E et al (2015) Characteristic CSF prion seeding efficiency in humans with prion diseases. Mol Neurobiol 51(1):396-405

23. De Luca CMG, Elia AE, Portaleone SM, Cazzaniga FA, Rossi M, Bistaffa E et al (2019) Efficient RT-QulC seeding activity for a-Synuclein in olfactory mucosa samples of patients with Parkinson's disease and multiple system atrophy. Transl Neurodegener 8:24

24. Dickson DW (2012) Parkinson's disease and parkinsonism: neuropathology. Cold Spring Harb Perspect Med 2(8):a009258

25. Dickson DW, Fujishiro H, DelleDonne A, Menke J, Ahmed Z, Klos KJ et al (2008) Evidence that incidental Lewy body disease is pre-symptomatic Parkinson's disease. Acta Neuropathol 115(4):437-444

26. Dujardin S, Hyman BT (2019) Tau prion-like propagation: state of the art and current challenges. Adv Exp Med Biol 1184:305-325

27. Fairfoul G, McGuire LI, Pal S, Ironside JW, Neumann J, Christie S et al (2016) Alpha-synuclein RT-QuIC in the CSF of patients with alphasynucleinopathies. Ann Clin Transl Neurol 3(10):812-818

28. Fearnley JM, Lees AJ (1991) Ageing and Parkinson's disease: Substantia Nigra regional selectivity. Brain 114(Pt. 5):2283-2301

29. Fiorini M, Iselle G, Perra D, Bongianni M, Capaldi S, Sacchetto L et al (2020) High diagnostic accuracy of RT-QulC assay in a prospective study of patients with suspected SCJD. Int J Mol Sci 21(3):880

30. Foutz A, Appleby BS, Hamlin C, Liu X, Yang S, Cohen Y et al (2017) Diagnostic and prognostic value of human prion detection in cerebrospinal fluid. Ann Neurol 81(1):79-92

31. Franceschini A, Baiardi S, Hughson AG, McKenzie N, Moda F, Rossi M et al (2017) High diagnostic value of second generation CSF RT-QulC across the wide spectrum of CJD prions. Sci Rep 7(1):10655

32. Garrido A, Fairfoul G, Tolosa ES, Martí MJ, Green A, Barcelona LRRK2 Study Group (2019) a-synuclein RT-QulC in cerebrospinal fluid of LRRK2-linked Parkinson's disease. Ann Clin Transl Neurol 6(6):1024-1032

33. Gelpi E, Navarro-Otano J, Tolosa E, Gaig C, Compta Y, Rey MJ et al (2014) Multiple organ involvement by alpha-synuclein pathology in Lewy body disorders. Mov Disord 29(8):1010-1018

34. Gilman S, Wenning GK, Low PA, Brooks DJ, Mathias CJ, Trojanowski JQ et al (2008) Second consensus statement on the diagnosis of multiple system atrophy. Neurology 71(9):670-676

35. Greffard S, Verny M, Bonnet A, Beinis J, Gallinari C, Meaume S et al (2006) Motor score of the unified Parkinson disease rating scale as a good predictor of Lewy body-associated neuronal loss in the Substantia Nigra. Arch Neurol 63(4):584-588

36. Groveman BR, Orrú CD, Hughson AG, Bongianni M, Fiorini M, Imperiale D et al (2016) Extended and direct evaluation of RT-QulC assays for Creutzfeldt-Jakob disease diagnosis. Ann Clin Transl Neurol 4(2):139-144

37. Groveman BR, Orrù CD, Hughson AG, Raymond LD, Zanusso G, Ghetti $B$ et al (2018) Rapid and ultra-sensitive quantitation of disease-associated asynuclein seeds in brain and cerebrospinal fluid by aSyn RT-QulC. Acta Neuropathol Commun 6(1):7

38. Hayashi Y, Iwasaki Y, Yoshikura N, Asano T, Mimuro M, Kimura A et al (2017) An autopsy-verified case of steroid-responsive encephalopathy with convulsion and a false-positive result from the real-time quaking-induced conversion assay. Prion 11(4):284-292

39. Henderson MX, Sengupta M, Trojanowski JQ, Lee VMY (2019) Alzheimer's disease tau is a prominent pathology in LRRK2 Parkinson's disease. Acta Neuropathol Commun 7(1):183

40. Högl B, Stefani A, Videnovic A (2018) Idiopathic REM sleep behaviour disorder and neurodegeneration - an update. Nat Rev Neurol 14(1):40-55

41. Holec SAM, Woerman AL (2020) Evidence of distinct a-Synuclein strains underlying disease heterogeneity. Acta Neuropathol. https://doi.org/10. 1007/s00401-020-02163-5

42. Iranzo A, Fairfoul G, Ayudhaya N, Chumbala A, Serradel M, Gelpi E et al (2020) Cerebrospinal fluid a-Synuclein detection by RT-QulC in patients with isolated rapid-eye-movement sleep behaviour disorder. https://doi. org/10.2139/ssrn.3556635 Available at SSRN: https://ssrn.com/abstract= 3556635

43. Jaunmuktane $Z$, Brandner S (2019) Invited review: the role of prion-like mechanisms in neurodegenerative diseases. Neuropathol Appl Neurobiol. https://doi.org/10.1111/nan.12592 
44. Kang UJ, Boehme AK, Fairfoul G, Shahnawaz M, Ma TC, Hutten SJ et al (2019) Comparative study of cerebrospinal fluid a-Synuclein seeding aggregation assays for diagnosis of Parkinson's disease. Mov Disord 34(4): 536-544

45. Kang UJ, Goldman JG, Alcalay RN, Xie T, Tuite P, Henchcliffe C et al (2016) The BioFIND study: characteristics of a clinically typical Parkinson's disease biomarker cohort. Mov Disord 31(6):924-932

46. Kaufmann H, Norcliffe-Kaufmann L, Palma JA, Biaggioni I, Low PA, Singer W, Autonomic Disorders Consortium et al (2017) Natural history of pure autonomic failure: a United States prospective cohort. Ann Neurol 81(2): 287-297

47. Kocisko DA, Come HJ, Priola SA, Chesebro B, Raymond GJ, Lansbury PT et al (1994) Cell-free formation of protease-resistant prion protein. Nature 370(6489):471-474

48. Koga S, Aoki N, Uitti RJ, van Gerpen JA, Cheshire WP, Josephs KA et al (2015) When DLB, PD, and PSP masquerade as MSA: an autopsy study of 134 patients. Neurology 85(5):404-412

49. Kraus A, Saijo E, Metrick MA 2nd, Newell K, Sigurdson CJ, Zanusso G et al (2019) Seeding selectivity and ultrasensitive detection of tau aggregate conformers of Alzheimer disease. Acta Neuropathol 137(4):585-598

50. Lattanzio F, Abu-Rumeileh S, Franceschini A, Kai H, Amore G, Poggiolini I et al (2017) Prion-specific and surrogate CSF biomarkers in Creutzfeldt-Jakob disease: diagnostic accuracy in relation to molecular subtypes and analysis of neuropathological correlates of p-tau and A $\beta 42$ levels. Acta Neuropathol 133(4):559-578

51. Mammana A, Baiardi S, Rossi M, Franceschini A, Donadio V, Capellari S et al (2020) Detection of prions in skin punch biopsies of Creutzfeldt-Jakob disease patients. Ann Clin Transl Neurol 7(4):559-564

52. Manne S, Kondru N, Hepker M, Jin H, Anantharam V, Lewis M et al (2019) Ultrasensitive detection of aggregated a-Synuclein in glial cells, human cerebrospinal fluid, and brain tissue using the RT-QulC assay: new highthroughput Neuroimmune biomarker assay for Parkinsonian disorders. J Neurolmmune Pharmacol 14(3):423-435

53. Martí-Massó JF, Ruiz-Martínez J, Bolaño MJ, Ruiz I, Gorostidi A, Moreno F et al (2009) Neuropathology of Parkinson's disease with the R1441G mutation in LRRK2. Mov Disord 24(13):1998-2001

54. McGuire LI, Peden AH, Orrú CD, Wilham JM, Appleford NE, Mallinson G et al (2012) Real time quaking-induced conversion analysis of cerebrospinal fluid in sporadic Creutzfeldt-Jakob disease. Ann Neurol 72(2):278-285

55. McGuire LI, Poleggi A, Poggiolini I, Suardi S, Grznarova K, Shi S et al (2016) Cerebrospinal fluid real-time quaking-induced conversion is a robust and reliable test for sporadic Creutzfeldt-Jakob disease: an international study. Ann Neurol 80(1):160-165

56. McKinley MP, Bolton DC, Prusiner SB (1983) A protease-resistant protein is a structural component of the scrapie prion. Cell 35(1):57-62

57. Metrick MA 2nd, Ferreira NDC, Saijo E, Kraus A, Newell K, Zanusso G et al (2020) A single ultrasensitive assay for detection and discrimination of tau aggregates of Alzheimer and pick diseases. Acta Neuropathol Commun 8(1):22

58. Miki Y, Foti SC, Asi YT, Tsushima E, Quinn N, Ling H et al (2019) Improving diagnostic accuracy of multiple system atrophy: a clinicopathological study. Brain 142(9):2813-2827

59. Orrú CD, Bongianni M, Tonoli G, Ferrari S, Hughson AG, Groveman BR et al (2014) A test for Creutzfeldt-Jakob disease using nasal brushings. N Engl J Med 371(6):519-529

60. Orrú CD, Groveman BR, Hughson AG, Zanusso G, Coulthart MB, Caughey B (2015) Rapid and sensitive RT-QulC detection of human Creutzfeldt-Jakob disease using cerebrospinal fluid. mBio 6(1):e02451-e02414

61. Orrú CD, Yuan J, Appleby BS, Li B, Li Y, Winner D et al (2017) Prion seeding activity and infectivity in skin samples from patients with sporadic Creutzfeldt-Jakob disease. Sci Transl Med 9(417):eaam7785

62. Parchi P, Capellari S (2013) Prion disease: diagnostic value of cerebrospinal fluid markers. Nat Rev Neurol 9(1):10-11

63. Parchi $P$, de Boni $L$, Saverioni $D$, Cohen $M L$, Ferrer I, Gambetti $P$ et al (2012) Consensus classification of human prion disease histotypes allows reliable identification of molecular subtypes: an inter-rater study among surveillance centres in Europe and USA. Acta Neuropathol 124(4):517-529

64. Parchi P, Strammiello R, Notari S, Giese A, Langeveld JP, Ladogana A et al (2009) Incidence and spectrum of sporadic Creutzfeldt-Jakob disease variants with mixed phenotype and co-occurrence of PrPSc types: an updated classification. Acta Neuropathol 118(5):659-671
65. Parchi P, Giese A, Capellari S, Brown P, Schulz-Schaeffer W, Windl O et al (1991) Classification of sporadic Creutzfeldt-Jakob disease based on molecular and phenotypic analysis of 300 subjects. Ann Neurol 46(2):224233

66. Postuma RB, Berg D, Stern M, Poewe W, Olanow CW, Oertel W et al (2015) MDS clinical diagnostic criteria for Parkinson's disease. Mov Disord 30(12): $1591-1601$

67. Postuma RB, Poewe W, Litvan I, Lewis S, Lang AE, Halliday G et al (2018) Validation of the MDS clinical diagnostic criteria for Parkinson's disease. Mov Disord 33(19):1601-1608

68. Prusiner SB (1982) Novel proteinaceous infectious particles cause scrapie. Science 216(4542):36-144

69. Prusiner SB (1998) Prions. Proc Natl Acad Sci U S A 95(23):13363-13383

70. Prusiner SB, Groth DF, Bolton DC, Kent SB, Hood LE (1984) Purification and structural studies of a major scrapie prion protein. Cell 38(1):127-134

71. Rey NL, Steiner JA, Maroof N, Luk KC, Madaj Z, Trojanowski JQ et al (2016) Widespread Transneuronal propagation of a-Synucleinopathy triggered in olfactory bulb mimics prodromal Parkinson's disease. J Exp Med 213(9): 1759-1778

72. Rhoads DD, Wrona A, Foutz A, Blevins J, Glisic K, Person M et al (2020) Diagnosis of prion diseases by RT-QulC results in improved surveillance. Neurology. https://doi.org/10.1212/WNL.0000000000010086

73. Rizzo G, Arcuti S, Copetti M, Alessandria M, Savica R, Fontana A et al (2018) Accuracy of clinical diagnosis of dementia with Lewy bodies: a systematic review and meta-analysis. J Neurol Neurosurg Psychiatry 89(4):358-366

74. Rossi M, Candelise N, Baiardi S, Capellari S, Giannini G, Orrù CD et al (2020) Ultrasensitive RT-QuIC assay with high sensitivity and specificity for Lewy body-associated synucleinopathies. Acta Neuropathol. https://doi.org/10. 1007/s00401-020-02160-8

75. Rudge P, Hyare H, Green A, Collinge J, Mead S (2018) Imaging and CSF analyses effectively distinguish CJD from its mimics. J Neurol Neurosurg Psychiatry 89(5):461-466

76. Saborio GP, Permanne B, Soto C (2001) Sensitive detection of pathological prion protein by cyclic amplification of protein misfolding. Nature 411(6839):810-813

77. Safar J, Roller PP, Gajdusek DC, Gibbs CJ Jr (1993) Thermal stability and conformational transitions of scrapie amyloid (prion) protein correlate with infectivity. Protein Sci 2(12):2206-2216

78. Saijo E, Ghetti B, Zanusso G, Oblak A, Furman JL, Diamond Ml et al (2017) Ultrasensitive and selective detection of 3-repeat tau seeding activity in pick disease brain and cerebrospinal fluid. Acta Neuropathol 133(5):751-765

79. Saijo E, Metrick MA 2nd, Koga S, Parchi P, Litvan I, Spina S et al (2020) 4-repeat tau seeds and templating subtypes as brain and CSF biomarkers of frontotemporal lobar degeneration. Acta Neuropathol Commun 139(1):63-77

80. Sanders DW, Kaufman SK, DeVos SL, Sharma AM, Mirbaha H, Li A et al (2014) Distinct tau prion strains propagate in cells and mice and define different Tauopathies. Neuron 82(6):1271-1288

81. Sano K, Satoh K, Atarashi R, Takashima H, Iwasaki Y, Yoshida M et al (2013) Early detection of abnormal prion protein in genetic human prion diseases now possible using real-time QUIC assay. PLOS One 8(1):e54915. https://doi. org/10.1371/journal.pone

82. Shahnawaz M, Mukherjee A, Pritzkow S, Mendez N, Rabadia P, Liu X et al (2020) Discriminating a-Synuclein strains in Parkinson's disease and multiple system atrophy. Nature 578(7794):273-277

83. Shahnawaz M, Tokuda T, Waragai M, Mendez N, Ishii R, Trenkwalder C et al (2017) Development of a biochemical diagnosis of Parkinson disease by detection of a-Synuclein Misfolded aggregates in cerebrospinal fluid. JAMA Neurol 74(2):163-172

84. Shiga Y, Miyazawa K, Sato S, Fukushima R, Shibuya S, Sato Y et al (2004) Diffusion-weighted MRI abnormalities as an early diagnostic marker for Creutzfeldt-Jakob disease. Neurology 63(3):443-449

85. Simrén J, Ashton NJ, Kaj Blennow K, Zetterberg H (2020) An update on fluid biomarkers for neurodegenerative diseases: recent success and challenges ahead. Curr Opin Neurobiol 61:29-39

86. Soto C, Anderes L, Suardi S, Cardone F, Castilla J, Frossard MJ et al (2005) Pre-symptomatic detection of prions by cyclic amplification of protein misfolding. FEBS Lett 579(3):638-642

87. Spillantini MG, Schmidt ML, Lee VM, Trojanowski JQ, Jakes R, Goedert M (1997) Alpha-synuclein in Lewy bodies. Nature 388(6645):839-840 
88. Vallabh SM, Minikel EV, Williams VJ, Carlyle BC, McManus AJ, Wennick CD (2020) Cerebrospinal fluid and plasma biomarkers in individuals at risk for genetic prion disease. BMC Med 18(1):140

89. van Rumund A, Green AJE, Fairfoul G, Esselink RAJ, Bloem BR, Verbeek MM

(2019) a-Synuclein real-time quaking-induced conversion in the cerebrospinal fluid of uncertain cases of parkinsonism. Ann Neurol 85(5):777-781

90. Vitali P, Maccagnano E, Caverzasi E, Henry RG, Haman A, Torres-Chae C et al (2011) Diffusion-weighted MRI hyperintensity patterns differentiate CJD from other rapid dementias. Neurology 76(20):1711-1719

91. Wilham JM, Orrú CD, Bessen RA, Atarashi R, Sano K, Race B et al (2010) Rapid end-point quantitation of prion seeding activity with sensitivity comparable to bioassays. PLoS Pathog 6(12):e1001217

92. Young GS, Geschwind MD, Fischbein NJ, Martindale JL, Henry RG, Liu S et al (2005) Diffusion-weighted and fluid-attenuated inversion recovery imaging in Creutzfeldt-Jakob disease: high sensitivity and specificity for diagnosis. AJNR Am J Neuroradiol 26(6):1551-1562

93. Zerr I, Kallenberg K, Summers DM, Romero C, Taratuto A, Heinemann U et al (2009) Updated clinical diagnostic criteria for sporadic Creutzfeldt-Jakob disease. Brain 132(Pt. 10):2659-2668

94. Zerr I, Parchi P (2018) Sporadic Creutfeldt-Jakob disease. Handb Clin Neurol 153:155

\section{Publisher's Note}

Springer Nature remains neutral with regard to jurisdictional claims in published maps and institutional affiliations.

Ready to submit your research? Choose BMC and benefit from:

- fast, convenient online submission

- thorough peer review by experienced researchers in your field

- rapid publication on acceptance

- support for research data, including large and complex data types

- gold Open Access which fosters wider collaboration and increased citations

- maximum visibility for your research: over $100 \mathrm{M}$ website views per year

At BMC, research is always in progress.

Learn more biomedcentral.com/submissions 